\title{
¿En qué consiste un docente con pensamiento crítico? Caso del Politécnico Colombiano Jaime Isaza Cadavid-Colombia ${ }^{1}$
}

\author{
Alfredo Enrique Zambrano Berdugo ${ }^{2}$ \\ Edgar Correa López ${ }^{3}$ \\ Henri Helí González Gaitán ${ }^{4}$ \\ Juan Horacio Botero Álvarez ${ }^{5}$
}

\section{Resumen}

La finalidad de este trabajo de investigación es identificar, indagar, conocer, definir, estructurar y ampliar los horizontes sobre el significado de un ser humano que se dedique a la enseñanza y a sus prácticas pedagógicas, la trascendencia e importancia que tiene para desarrollar una labor fructífera y eficiente, el obtener un criterio y una visión en su labor, la cual esté mediada por actuar con pensamiento crítico. Se intuye que este atributo del pensamiento, permite que la labor pedagógica sea más analítica, más vivencial, más estructurada, más significativa y, por lo tanto, más adecuada a las necesidades y perspectivas de las personas que aprenden, los estudiantes.

La metodología que utilizamos para lograr este cometido, fue esencialmente a través de nuestras propias autobiografías, o correlatos, porque consideramos que no tiene sentido hablar de que otras personas posean pensamiento crítico o no, si nosotros no nos cuestionamos sobre la existencia del mismo y sobre la manera como

1 Recibido: 30 de noviembre de 2017. Aceptado: 20 de junio de 2018.

2 Alfredo Enrique Zambrano Berdugo. Magister en Educación Docencia. Docente Universitario. Licenciado en Educación. Especialidad en Ciencias Sociales y Economía. Universidad del Atlántico. Correo Electrónico: alzabe6319@hotmail.com

3 Edgar Correa López. Docente Universitario. Especialista en Gerencia Integral, Politécnico Jaime Isaza Cadavid. Administrador de Empresas, UNAD. Tecnólogo Industrial Politécnico Jaime Isaza Cadavid. Magister en Educación Docencia. Universidad de Manizales. Correo Electrónico: ecoloys@yahoo.es

4 Henry Helí González Gaitán. Magister en Educación Docencia. Docente universitario. Ingeniero Industrial, Universidad de Antioquia. Especialista en Logística Integral, U de A y Gestión Energética Industrial, ITM. Correo Electrónico: henryheligg@gmail.com

5 Juan Horacio Botero Alvarez. Magister en Educación Docencia. Docente universitario. Psicólogo Clínico, Universidad Antonio Nariño. Especialista en Selección de Personal, Universidad Complutense, Madrid, España. Correo Electrónico: jhoracio@une.net.co 
ha permeado nuestras vidas. En este trabajo de investigación, los participantes, somos al mismo tiempo, los sujetos investigadores, como los sujetos investigados. Para lograr conseguir este objetivo, cada uno de los integrantes de esta investigación, realizó un análisis desde sus vivencias, la aplicación que hubiera tenido durante su vida, en sus actuaciones tanto pedagógicas como cotidianas, la trayectoria y disposición con pensamiento crítico. Queremos llegar a establecer una conclusión que nos permita pensar y obrar de manera diferente, porque no queremos seguir actuando de la misma forma, como lo hemos venido haciendo, pues esto nos impide que nos adaptemos a los cambios que exige el momento actual, como son la tecnología y la globalización.

Palabras clave: Pensamiento, crítico, significado, discernimiento, visión, pedagogía, quehacer educativo.

\section{¿What does a teacher with critical thinking consist on? Case study by Polytechnic Colombian Jaime Isaza Cadavid-Colombia}

\section{Abstract}

The aim this research is about to identify, investigate, know, define, organize and widen limits over the meaning of that human being who works for teaching and his/her pedagogical practices; and transcendence and importance that meaning has in development of a good and efficient job in order to obtain an opinion and a vision ruled by a critical thought behavior. It suggests this attribute of the thinking permits that teachers' pedagogical work can be more analytic, more practical, more structured and, more meaningful and, therefore more suitable with needs and career prospective from those who learn: The students.

The methodology we used to achieve this task was essentially through our own autobiographies, or correlates, because we believe that it does not make sense to talk about other people having critical thinking or not, if we do not question the existence of it and about the way he has permeated our lives. In this research work, the participants are, at the same time, the research subjects, as the subjects investigated. To achieve this goal, each of the members of this research, made an analysis from their experiences, the application that would have had during his life, in his actions both pedagogical and daily, the trajectory and disposition with critical thinking. We want to reach a conclusion that allows us to think and act diffe- 
rently, because we do not want to continue acting in the same way, as we have been doing, because this prevents us from adapting to the changes required by the current time, such as technology and globalization.

Key words: Critical, thinking, meaning, discernment, vision, pedagogy, educational work.

\section{Preámbulo}

\begin{abstract}
"Un docente con pensamiento crítico duda en forma constante de lo que sabe, no busca imponer verdades y debilita sus propios dogmas y paradigmas...". Miguel Alberto González González. (2014).
\end{abstract}

Nuestra condición humana, se ha aferrado a un modernismo "crítico" pero sobre todo, basado más en el orgullo y el egoísmo que se incentiva desde la escuela con reconocimientos públicos desde el individualismo, sin tener criterios propios y auténticos, que nos permitan ser más desde lo humano, que del conocimiento y el pensamiento crítico. El docente hoy, es un sujeto moderno, cree que: "es más por lo que sabe, que por lo que es y no por lo que pueda llegar a pensar". (Botero, Correa, González, Zambrano, 2014).

La modernidad dentro de lo humano es muy diferente, lleva a la formación de la persona desde una perspectiva dimensional única, que lo convierte en un ser social que piensa, que avanza, que define su propio cuestionamiento, su propia filosofía de vida, que lo va a caracterizar desde lo más hondo de su entorno social que lo afecta.

Ser docente hoy, implica una gran función dentro de la escala social a ni- vel de sujeto-objeto, que nos permita entender la clase de estudiantes que deseamos salgan de nuestras aulas de clases. Estudiantes que no traguen "entero", que se inclinen por la funcionalidad del ser en cuanto a su pensar, antes que a la irracionalidad de los otros en su formar de actuar. El estudiante que queremos, debe salir con pautas claras, propuestas por el docente con un pensamiento crítico, independiente, que se haga sentir y notar por encima de cualquier sistema que vaya en contra de lo que realmente quiere lograr como sujeto, que no le dé miedo expresar lo que siente, y hacer lo que realmente desea, no lo que el otro le imponga.

La de-colonización es un aspecto histórico que se aborda como fundamento filosófico del nuevo docente con pensamiento crítico, capaz de entender el contexto del entorno y participar en el aula en los procesos de enseñanza aprendizaje, en el cual, con el estudiante, se construya día a día, la propia historia, la historia de cada uno, y no la que nos relata el espectador.

A esto, debemos invitar a nuestros estudiantes que desde su presente, construyan su propia historia. Que desde su realidad y postura, entien- 
Volumen 22 Nro.1. Julio-Diciembre 2018

dan la necesidad de construir un pensamiento crítico, que los encamine a una realidad diferente. Dentro de nuestro contexto académico y pedagógico como docentes, debemos aprender a construir nuevos horizontes que nos permitan y le permitan al estudiante un mejor enfoque conceptual en cuanto a nuestra forma de pensar críticamente. Pensar críticamente, consiste en no quedarse solamente con el concepto, o la duda que nos generen, consiste en trascender, ir más allá de nuestra manera tradicional de pensar y actuar, pensar crítico me hace diferente y me amplía los horizontes del conocimiento.

\section{Investigaciones precedentes}

Quisimos darnos a la tarea de investigar qué se ha dicho o hablado sobre el pensamiento crítico, teniendo en cuenta que no somos los primeros, ni los únicos en hablar, discutir, o proponer algo sobre el contenido del pensamiento crítico y esperamos que este estudio nos aporte nuevas ideas sobre el significado del mismo, aplicado al campo educativo, pedagógico y docente.

Nos parece conveniente aclarar que en el título del proyecto, se quiso hacer la aplicación de los principios, conceptos e ideas manifestadas en este trabajo, a la Institución Universitaria Politécnico Colombiano Jaime Isaza Cadavid, porque en dicha Institución laboramos los integrantes del equipo de trabajo, pero también podría aplicarse a otras Universidades, o a otros contextos educativos, pues adoptar un pensamiento crítico, no es necesario solo en el contexto universitario, sino desde que se inicia la formación de una persona, en pre-escolar, primaria, o bachillerato.

El Politécnico Colombiano Jaime Isaza Cadavid es una Institución Universitaria, ubicada en la ciudad de Medellín, departamento de Antioquia, Colombia, que se ha distinguido en su historia académica, por ofrecer carreras tecnológicas, pero también tiene carreras del ciclo profesional, con duración de 5 años, como la Ingeniería de Productividad y Calidad, Ingeniería Informática, Contaduría Pública, Profesional en Deportes y Profesional en Ciencias Agrarias.

En este año 2014, celebró sus 50 años de funcionamiento: posee tres sedes, la principal en el barrio el Poblado de Medellín y las otras dos en Rionegro y Apartadó (municipios ambos del departamento de Antioquia). Algunas de las carreras tecnológicas que ofrece son: Tecnología Industrial, Tecnología en Costos y Auditoría, Tecnología en Administración Financiera, Tecnología Aeroportuaria, Tecnología Logística y Tecnología en Instrumentación Industrial.

Una persona que se dedique a la enseñanza como docente, le resulta conveniente, provechoso y práctico que sus estudiantes tengan una forma de pensar crítica, porque quieran analizar, profundizar, discutir y cuestionar los conocimientos que van aprendiendo con sus docentes, en el aula de clase y también fuera de ella. Con el fin de que los estudiantes adquieran y utilicen el pensamiento crítico, se necesita que la comunicación 
entre el docente y los estudiantes sea fluida, sincera, abierta y con un sentido de confianza y sinceridad entre ambos sujetos.

Consultamos unas tesis de grado que se refieren al pensamiento crítico y que nos permitieron tener una mayor aproximación a su significado, sus características, los medios o herramientas para adquirirlo, las posibles dificultades en su apropiación, su estructura y sus formas de aplicación. Esto nos permitió sensibilizarnos más en su adquisición y conveniencia en su utilización.

Es necesario especificar que nuestro trabajo de investigación está orientado a conceptualizar y caracterizar una serie de ideas y proposiciones que sean viables para el ámbito educativo-docente, las cuales obtuvimos a partir de nuestras autobiografías, o correlatos, del contacto con teóricos de la Escuela de Frankfurt, como Adorno, Marcuse y Habermas, también de Peter McLaren, que sin ser específicamente de la Escuela, aportó ideas relevantes sobre la teoría crítica y la revolucionaria y todos ellos realizaron propuestas y alusiones al tema del pensamiento crítico. Todo esto nos permitió conocer más a fondo el significado del pensar crítico y hacer sugerencias que contribuyan a promover el bienestar y el mejoramiento de la calidad educativa, no solo en Colombia, sino a nivel internacional.

Por lo tanto, nosotros como sujetos investigadores, partimos más de nosotros mismos para realizar el proyecto y ofrecer proyecciones, que tener propuestas o sugerencias de otros docentes, o de algunos estudiantes.
Una de las tesis consultada se denominaba: "Herramientas cognitivas para desarrollar el pensamiento crítico en estudiantes de la formación inicial docente" realizada por el Sr. Eduardo González Guzmán, en la Universidad de San Carlos de Guatemala, en Noviembre de 2010, para optar al título de Doctor en Filosofía y Educación.

Esta tesis, consistió en desarrollar un proyecto educativo que permita identificar cómo se articula en la práctica, el desarrollo y evolución del pensamiento crítico en estudiantes de la Universidad antes mencionada. La finalidad de la educación y de los educadores, es estimular el pensamiento crítico de los estudiantes y ofrecer posibilidades de desarrollo y expresión, con el fin de lograr un aprendizaje significativo. Uno de los principales elementos del pensamiento crítico, son las herramientas cognitivas, porque permiten visualizar los problemas y desarrollar patrones lógicos de pensamiento.

Otra de las tesis consultada se denominaba: "Pensamiento crítico: diferencias en estudiantes universitarios en el tipo de creencias, estrategias e inferencias en la lectura crítica de textos" realizada por Gloria Patricia Marciales Vivas de la Universidad Complutense de Madrid, España, 2003. El objetivo de esta tesis fue mostrar la influencia del pensamiento crítico para leer textos, sacar conclusiones y hacer inferencias.

En este trabajo, también se abordó la calidad de la educación, teniendo en cuenta la crisis por la que atravie- 
Volumen 22 Nro.1. Julio-Diciembre 2018

san la mayoría de las Universidades del mundo. La calidad de la educación es un concepto multidimensional, que incluye todas las funciones y actividades de la Universidad, Colegio o Escuela. Se debe proponer un modelo de educación centrado en el estudiante, el cual debe estar orientado a obtener los conocimientos de las diferentes disciplinas curriculares para desarrollar competencias, aptitudes para la comunicación, la reflexión independiente y el trabajo en equipo.

La otra tesis se denominaba: "El pensamiento lógico desde la perspectiva de las Neurociencias Cognitivas" presentada por Rafael Blanco Menéndez de la Universidad de Oviedo, España, Octubre 2008, para obtener el Doctorado en Neuropsicología Cognitiva.

La finalidad de esta tesis fue mostrar la influencia que poseen las neurociencias cognitivas, neurobiológicas y neurofisiológicas en los procesos de pensamiento lógico y crítico de las personas. Los procesos lógicos de pensamiento se abordan en esta tesis desde una perspectiva asociacionista-conductista.

Cada una de estas tesis, nos condujo a plantearnos: los estudiantes deben ser iniciados desde unas perspectivas totalmente diferentes, saliéndose del sistema parametral-conductista, al que hemos estado sometidos desde nuestros inicios académicos. Los investigadores nos dan pautas significativas para nuestro trabajo, porque nos permiten identificar que a través del lenguaje, el estudiante es capaz de generar nuevos enfoques en su manera de pensar y asimilar el conocimiento.

\section{Situación problematizadora}

Para definir el problema a investigar, necesitamos expresar los aspectos que hemos detectado a través de la observación y del dialogo con otros docentes, sobre los comentarios que realizan acerca de la metodología que utilizan en la realización de sus prácticas pedagógicas: se percibe una forma tradicional de enseñanza, la cual se caracteriza por la repetición de lo que se ha hecho siempre, pues les ha dado buen resultado. No se observa un sentido de transformación educativa, cultural y social, que tenga impacto en la vida académica y que se adapte a los paradigmas que nos ofrece la sociedad de hoy, como son la tecnología y la globalización.

Ante esta situación $y$, teniendo en cuenta que a muchos docentes poco les interesa adoptar en su trabajo pedagógico una herramienta que les ayude a mejorar su labor, como sería el pensar crítico, porque simplemente no quieren salir de su zona de confort y porque esto les exige mayor tiempo de dedicación, mayor análisis en la presentación de los temas y currículos, reflexión sobre las ventajas y desventajas que signifique el cambio, poco grado de aceptación por parte de los estudiantes que no están dispuestos a cambiar el paradigma educativo tradicional. Entonces, el problema a investigar, es;

Teniendo en cuenta estas observaciones, definimos el problema como lo expresamos al comienzo de este trabajo: 
“¿En qué consiste un docente con pensamiento crítico? Caso del Politécnico Colombiano Jaime Isaza Cadavid?"

Mencionamos a continuación, algunos fragmentos de los correlatos que realizamos los investigadores, los cuales nos aportaron para llegar a la pregunta de investigación, con el fin que el lector los conozca y se perciba una justificación del problema a investigar:

Uno de los investigadores comenta en su correlato que "es de vital importancia la observación del proceso, con la aplicación de una Pedagogía crítica y una didáctica coherente, que responda al contexto. Al respecto, debemos tener presente siempre en la enseñanza, las emociones". González, Henry (2014).

Otro investigador afirma:

"en otras palabras, podemos decir que la propuesta del docente está orientada a tener una mirada retrospectiva que permita mostrar sus influencias en el presente y obtener una mirada diferente de la vida, los hechos y acontecimientos que se vivan, sin perder el enfoque $u$ objetivo del trabajo investigativo". Botero, (2014).

En la misma dirección, otro de los investigadores expresa en su correlato, manifestando la evidencia de sus miedos: "si en el salón de clases, en segundo año de primaria, no sabía responder una pregunta, me ponía a llorar, no sé describirlo con detalles, pero sé que pasó algunas veces, aún recuerdo aquel salón". Correa, (2014).

Por último, un fragmento de otro de los investigadores, manifiesta: "en lo que respecta al pensamiento crítico y lo que siento hacia él, en el Bachillerato y en la Universidad, tanto a nivel de pregrados y especializaciones, he mantenido la constante de que las cosas se deben hacer de mejor forma, no me quedo por lo general con el concepto del profesor, trasciendo hacia una nueva posibilidad, de cómo sería aplicar de otra forma lo que el profesor explica, no desde lo tradicional o parametral". Zambrano, (2014).

\section{Horizontes de Formación}

"Lo más dinámico del pensador crítico es la duda profunda, el no creer verdades sus verdades". Miguel Alberto González González (2013)

Identificar en el entorno académico desde el análisis, los aspectos fundamentales de la Metacognición, episteme y las autobiografías o correlatos, en el contexto donde se desenvuelve un docente con pensamiento crítico.

Pro-poner desde la epistemología y las autobiografías, fundamentos al docente para el desarrollo de un pensamiento crítico y contribuir en la formación de profesionales integrales con sentido crítico, capaces de propiciar cambios en el entorno.

\section{Conversando con los teóricos, autores}

En éste capítulo, pretendemos mostrar que hemos escogido los principales autores que se refieren directa o indirectamente al pensamiento crítico, como son los de la Escuela de Frankfurt, porque a través de los supuestos que nos proporcionaron acerca de la Teoría Crítica, la evolución que ellos demostraron, refirién- 
Volumen 22 Nro.1. Julio-Diciembre 2018

dose a la situación que vivía la sociedad en ese entonces (1923-1940), los cuestionamientos que hicieron sobre los problemas sociales y la forma de abordarlos, nos permitieron ir construyendo una serie de ideas y principios sobre el verdadero significado del pensamiento crítico.

¿Pero cuáles son estos teóricos? Nos referimos específicamente a los representantes de la Escuela de Frankfurt, como Adorno, Horkheimer, Marcuse y Habermas, como también a Francisco Mora en sus planteamientos sobre la Neuro-educación, porque ellos a través de sus escritos, análisis, consideraciones y reflexiones, sentaron las bases esenciales para comprender no solo lo que debe ser el pensar crítico, sino las dificultades sociales de la época, que también podrían ser las nuestras, pero en otros contextos y otras problemáticas diferentes, lo cual nos permite construir nuestra propia historia.

En este trabajo es muy importante la intención, ya que esta implica una metodología de construcción de sujeto histórico, nuestra vida como sujeto social de historia. ¿Por qué somos maestros? ¿Cómo llegamos a ser maestros desde el presente para ver el pasado histórico y construir el futuro? Hay sucesos que son universales, pero hay especificidades históricas.

La Escuela de Frankfurt surge con la fundación, en 1923, del Instituto para la Investigación Social en Alemania. Es una conceptualización de la teoría de la sociedad que cuestiona el rol de la comunicación por la desigualdad en el poder y crea una industria cul- tural que alinea las mentes. Surgieron un conjunto de teorías que serían conocidas como la Teoría Crítica, que trascendieron del concepto de la Teoría Crítica a la Teoría Revolucionaria.

Estas teorías se difundieron con el fin de ampliar la mirada de las concepciones críticas de Hegel, Marx y Freud. Algunas obras como La Dialéctica del lluminismo y La Personalidad Autoritaria (Horkheimer) fueron conocidas e impulsadas en América. La muerte de Adorno (1969), y de Max Horkheimer (1973) dieron origen a la segunda generación de la Escuela de Frankfurt y dentro de ésta se encuentra Jürgen $\mathrm{Ha}$ bermas, quien hace aportes muy significativos sobre la acción comunicativa.

Estos diálogos con autores, sirven para enriquecer la respuesta de la pregunta de investigación, entendiendo que si un buen número de intelectuales dedican parte de su valioso tiempo a escribir de alguien, es porque esa es una fuente de sabiduría. "Los educadores que sostienen una pedagogía revolucionaria están obligados a enfrentar los efectos negativos del capitalismo global" McLaren (2007, p. 34).

Este pensamiento crítico se circunscribe como un riesgo que el docente con pensamiento crítico, lo asume como propio. Se ha hallado aquí, que comúnmente los docentes sacan sus propias conclusiones respecto al desempeño de los estudiantes. Se puede decir, que "la Pedagogía Crítica es el primer paso para pasar necesariamente a una Pedagogía Revolucionaria, que trae al escenario de la educación esos entornos donde se visualiza claramente". McLaren (2007, p. 56). 
Del mismo modo, hace claridad, sin descartar también "que quienes aún habiendo nacido en la pobreza y llegan a posiciones de poder, toman posiciones como blancos e identificándose con la ideología de los más favorecidos" McLaren (2007, p. 58). Con respecto a la transición entre la pedagogía crítica y la pedagogía revolucionaria, afirma que: "la clase no es el único prejuicio y reconocer aspectos tales como el género, la raza, la ideología, la religión y la orientación sexual como parte de la dinámica de opresión" McLaren (2007, p. 65).

En su libro titulado Neuro-educación, Francisco Mora, ilustra el título: "solo se puede aprender aquello que se ama" Mora (2013, carátula) y hemos aprendido a amar apasionadamente la Maestría en Educación-Docencia, desde el inicio de ella y nos hemos transformado en seres diferentes, con capacidad de observación y de entender mejor el proceso de Enseñanza-Aprendizaje. Debemos "aprovechar siempre lo mejor del conocimiento disponible para enseñar y aprender mejor" Mora (2013, p.13).

Por lo tanto, debemos despertar el amor por lo que enseñamos los docentes en el aula, necesitamos "conocer el cerebro para enseñar mejor" Mora (2013, p.15). Se trata, de descubrir y planificar mejores herramientas de enseñanza que faciliten el aprendizaje de las materias, o asignaturas que "detecten fallos psicológicos y cerebrales, que incapaciten para el normal aprendizaje, se promocione la empatía, el altruismo y la colaboración" Mora (2013, p. 17).
De tal manera, dice dar respuesta a un nuevo tiempo de reflexión, para lo cual desde la experiencia, cuando aprendíamos de docentes que utilizaban el Conductismo, llenando tableros y se pensaba: ¿no habrá una mejor forma de enseñar?, no había los adelantos que hoy los investigadores aportan desde el conocimiento mismo del cerebro, de tal manera que responda a la pregunta que formula: ¿"Qué hace que "un docente" se convierta en un docente excelente"? Mora (2013, p. 20).

Se plantea la pregunta:¿cómo los procesos mentales emergen de los procesos cerebrales?¿Cómo el cerebro aprende y memoriza, lo que sucede de modo acelerado? Para dar respuesta a estos interrogantes y a la pregunta de investigación ¿En qué consiste un docente con pensamiento crítico? Caso del Politécnico Colombiano Jaime Isaza Cadavid, se procede a identificar los conceptos más importantes y claves que deben considerarse en la práctica diaria del docente en el aula de clase.

En consecuencia, no basta con solicitarle al alumno que atienda, que aún sea activo o pasivo, hay que estimular o encender primero la emoción, para ello hay que utilizar métodos que se adapten a la alegría, despertar el placer y jamás el castigo, por eso "hoy sabemos que la letra con sangre no entra" Mora, (2013, p. 83).

En el momento actual solo debemos y podemos enseñar a través de la alegría, porque conocemos bien los sustratos cerebrales de estos procesos. 
Otra parte importante, en "el proceso de enseñanza-aprendizaje, son las emociones" Mora (2013, p. 66), porque sin ellas se vuelve más difícil el aprendizaje. Las emociones corresponden a otro cerebro dentro del cerebro, que se conoce como sistema límbico o cerebro emocional. "La emoción es la energía que mueve al mundo" Mora (2013, p. 68).

Aquí es donde los sujetos que intervienen el proceso de enseñanzaaprendizaje, especialmente el aprendiz, relacionan "los abstractos o ideas, con las que trabajan las cortezas de asociación para crear el pensamiento, ya están impregnadas de emoción" Mora (2013, p. 45).

Sin embargo, debe tenerse mucha claridad respecto a las emociones y los sentimientos, en el que están inmersos los sujetos Docente-Estudian- te, de tal manera que el entorno en que viven los sujetos actuantes, en el proceso de enseñanza-aprendizaje, usen su curiosidad epistémica en el descubrimiento de ese mundo que les rodea e impacta, de tal forma que motive al individuo a continuar observando e investigando. De lo anterior, se desprende que los docentes, debemos conocer estas manifestaciones de comportamiento en los estudiantes, una es, la forma de dirigirnos a ellos con frases que afectan o traen recordación negativa y no nos damos cuenta de estas afectaciones en sus vidas, en lo afectivo, social, familiar, entre otros, en el que los estudiantes se desenvuelven.

En el lenguaje que utilizamos para comunicarnos, Habermas propone cuatro fundamentos esenciales que son:

Figura 1: Fundamentos de Habermas. (Botero, Correa, González, Zambrano, 2014)

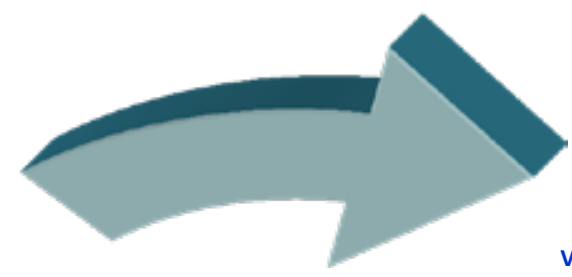

INTELIGIBILIDAD: que se comprenda lo que se dice

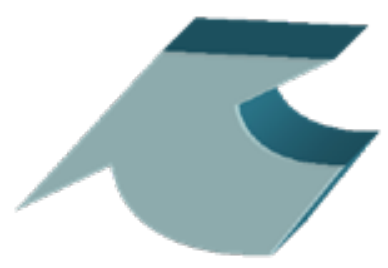

RECTITUD: se debe ser ético en lo que se dice

VERDADERO: que haya coherencia con la realidad.

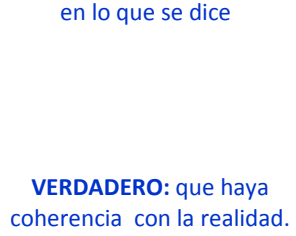

VERACIDAD: que siempre se diga la verdad.

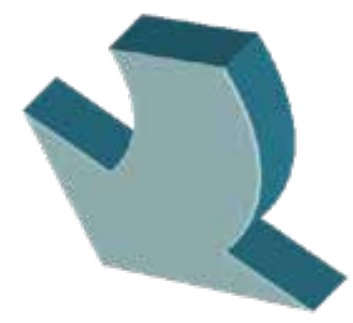

Fuente: elaboración propia 
Otro criterio de clasificación de las acciones para Habermas, en función del objetivo a conseguir en la comuni- cación, es el establecimiento de tres tipos de acción diferentes:

\section{Ilustración 2: Las acciones para Habermas}

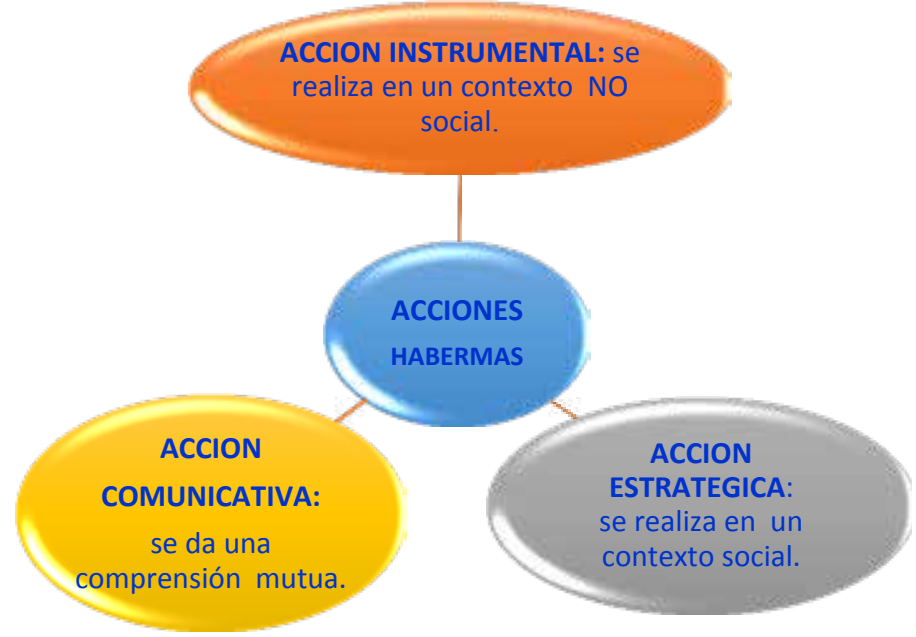

(Botero, Correa, González, Zambrano, 2014)

Habermas concibe tres clases o tipos de mundos, en su relación con los objetos, mundo físico, mundo de la conciencia y mundo objetivo. La Teoría Crítica es una teoría de la sociedad intelectual alemana, que cuestiona el rol de la comunicación en la desigualdad que se presenta en las personas que desean obtener un poder, con el fin de crear una industria cultural. Nunca se ha cuestionado la realidad de la vida y de las cosas por parte de la mayoría de los seres humanos, de ahí surge la teoría crítica, que se basa en el pensamiento crítico. La sociedad nos ha impuesto a los seres humanos una serie de condicionamientos que no nos dejan vivir como realmente debemos hacerlo.
"Los autores de la Escuela de Frankfurt consideran que la interacción social de los seres humanos, debe fundamentarse en el signo lingüístico, de tal forma que permita darle fuerza y esclarecer las verdades que son sometidas a la crítica". Habermas (1976, p.16)

Habermas presenta su teoría a partir de los supuestos universales del habla, lo cual significa que una persona, antes de decir cualquier cosa, se debe fundamentar en los mandatos del lenguaje. El interés social exige la renuncia al interés particular, porque es el que define al sistema, en un ordenamiento objetivo y científico de la sociedad. Habermas (1976, p.19).

Las acciones comunicativas son "las manifestaciones simbólicas en la 
Volumen 22 Nro.1. Julio-Diciembre 2018

cual el actor entra en relación con el mundo objetivo" Habermas (1976, p. 8). También se refiere al "mundo de la vida y al mundo de la acción comunicativa, como conceptos complementarios, porque la acción comunicativa ocurre dentro del mundo de la vida". Habermas (1976, p. 36).

Las ideas que nos han aportado estos autores, nos permiten darnos cuenta de que lo referente al pensamiento crítico, debe comenzar a adquirirse y fomentarse no solo desde la Escuela, sino también desde el hogar o la Familia y que el mundo de la vida debe desarrollarse y realizarse a través de la comunicación. Sin embargo, y por las características de nuestra investigación, acercamos estos desarrollos al marco de la educación superior, contexto objeto de nuestra intervención.

Al preguntarnos entonces: ¿ en qué consiste un docente con pensamiento crítico? Caso del Politécnico Colombiano Jaime Isaza Cadavid, y mejor aún, ¿cómo se puede lograr desde la condición de docente que nuestros estudiantes puedan formarse con pensamiento crítico?, sólo una cosa nos es clara hoy: itenemos que empezar por nosotros mismos los docentes! Nuestro trabajo de investigación recogió consideraciones acerca del pensamiento crítico, para ello, se requiere de un conjunto de habilidades cognitivas y disposiciones, como ingredientes fundamentales y necesarios para un buen pensador crítico.

Y en relación a estas necesidades, se citan entre otras, una comprensión epistemológica del pensamiento críti- co, el haber alcanzado un determinado nivel de desarrollo epistemológico, o las creencias que se mantienen sobre el pensamiento en sí mismo. Sobre este asunto, el texto Teoría de la Acción Comunicativa aporta elementos claros en dónde "el pensamiento crítico se materializa en el contexto diario, en las continuas posiciones reflexivas, donde se logra la construcción de sus propias conceptualizaciones personales y críticas" Habermas (1981,p. 18).

Un docente que en sus clases permanentemente lleve a sus estudiantes a asumir posiciones reflexivas sobre un tema, un libro, una actividad, una obra literaria o cualquier otro momento dentro del aula de clase, para llegar a una construcción conceptual clara y fuerte, sin duda alguna, es un docente que está apuntando a desarrollar pensamiento crítico en sus estudiantes.

No podemos olvidar que ésta, no es, ni será una tarea fácil, pero lo que sí es fácil, es que el docente se capacite, se auto cuestione frente a su quehacer diario para aportarle a su comunidad educativa todos los elementos requeridos para lograr estos desarrollos.

Muchos autores nos aportaron elementos valiosos en el trabajo de investigación en torno al pensamiento crítico, desde el aula de clase, pero hay en especial algunas concepciones que nos atrajeron la atención por la profundidad de sus posturas.

Theodor Adorno en su obra Dialéctica de la llustración pone de presente que "no entiende cómo es posible que 
la humanidad en vez de progresar, se esté hundiendo en la barbarie". Adorno (1968, p.106). Hace además, una serie de planteamientos interesantes frente al pensamiento crítico, determinado por el dominio de la naturaleza y afirma que con la Dialéctica de la Ilustración quiere realizar un análisis del concepto de razón, llegando a la conclusión de que en él, participan ideas de liberación y dominación.

A la dialéctica de la investigación, a la dialéctica de la negativa, o en esta última, menciona, recuperando una expresión Adorniana clave, esto significará para el pensamiento "decir lo que no se puede decir o, más aún, ir con el concepto más allá de él mismo". Adorno (1969, p.116). Como subraya:

"no hay posibilidad de un camino directo, esto es, de una intuición intelectual que salve inmediatamente la distancia entre el sujeto y lo otro del sujeto". Adorno (1969, p. 118).

Precisamente, no es posible porque el concepto, aunque instrumento de dominio, es también medio de rotura de la indiferenciación, esto es: el concepto es necesario pues en él se asienta a la vez la posibilidad de emancipación del sujeto, respecto del contexto natural absorbente.

Entonces parece claro que la alternativa de Adorno se moverá en una reformulación de la relación entre el pensamiento crítico y el concepto. $Y$ es completamente cierto y aplicable a la modernidad lo que dice Adorno, pues sin el pensamiento crítico no hay voz, no habría sujeto, ni naturaleza. Ante esta situación, el papel del intelectual de izquierda resulta profusamente cuestionado, pues, se veía ante la encrucijada del pensamiento crítico, libre de compromisos y la respuesta a un compromiso social,- político, que no comprometiera sus propuestas teóricas a favor de un partido.

Misión que la Escuela de Frankfurt tomó como propia y que encontró en la oferta filosófica de la época, donde trataba precisamente de la acción y la crítica del intelectual hacia el acercamiento teórico a la realidad. Parte importante de esta incursión en la teoría crítica de estos intelectuales de aquella época, se debió a esa capacidad de discernimiento, de pensamiento crítico, de deducción lógica, de entendimiento, de que su realidad no podía estar más lejos que su razón y fue por eso, que varios de ellos también decidieron salir exiliados de sus países, como fue el caso de Adorno, no solo por sus ideologías, sino por la influencia y crecimiento del nazismo en Alemania, lo que obligó a varios de sus representantes a huir a los Estados Unidos, donde se dedicaron a difundir sus teorías y principios.

Otro que tiene una postura importante con nuestra apuesta es Herbert Marcuse, en su postulado sobre el hombre unidimensional: inicia proponiendo nuevas alternativas sociales que lleven al individuo y a la sociedad por un camino diferente del que se ha tenido hasta ahora, como él mismo lo dice: "el individuo tendría libertad para ejercer la autonomía sobre una vida, que sería la suya propia". Marcuse (1954, p. 35). 
Volumen 22 Nro.1. Julio-Diciembre 2018

En su obra, Marcuse sugiere que "si el hombre tuviese la libertad intelectual, se retomaría el pensamiento individual, el cual, se encuentra absorbido por un consumismo exagerado y denigrante: el hombre por sus necesidades, vive sometido a ellas, pero a la vez, no son de gran cumplimiento sobre todo, aquellos oficios que le son impuestos por la sociedad para su represión, que lo someterán a vivir perpetuamente en la miseria y en la injusticia social" Marcuse (1954, p. 47).

La mayor parte de las necesidades predominantes, pertenecen a la categoría de falsas necesidades. En su afán y deseo de tener, poseer, obtener y satisfacer todas sus necesidades básicas, han convertido al sujeto en un ente completamente heterónimo, es decir, que el sujeto está sometido a un poder externo que le impide el libre desarrollo de su propia voluntad. El sujeto ha perdido la capacidad de pensar. Al no lograr reconocer cuáles son sus necesidades verdaderas o falsas, se le mantiene en un estado de adoctrinamiento y no es capaz de expresar su propia respuesta. "Bajo el gobierno de una totalidad represiva, la libertad se puede convertir en un poderoso instrumento de dominación" Marcuse (1954, p. 53).

Marcuse se refiere a los medios masivos de comunicación, los cuales encaminan al sujeto hacia un condicionamiento, que termina convirtiéndole en "esclavo" de sus propias necesidades y en una forma de vida totalmente represiva. Adelantado a su época, Marcuse ve con ojos de otra dimensión, el poder del control que ejerce y ejercerá la tecnología sobre la vida del ser humano, pretende dar pautas para vivir en un mundo mejor y ser críticos en nuestra forma de pensar: "mientras más capaz parezca la tecnología de crear las condiciones para la pacificación, más se organizan el espíritu y el cuerpo del hombre en contra de esta alternativa" Marcuse (1954, p. 67).

Aunque desde los orígenes de la sociedad, el hombre ha tenido los mismos derechos y libertades, a través de los tiempos, todo esto se ha perdido debido a las imposiciones a la que se ha visto sometido. Al respecto dice: "la libertad de pensamiento, de palabra y de conciencia, eran esencialmente ideas críticas, destinadas a reemplazar una cultura medioeval e intelectual anticuada, por otra más productiva y racional". Marcuse (1954, p. 85).

El consumismo sin control, en el que el hombre está sometido hoy, es lo que lo marca. Con lo anterior, volvemos nuevamente a la domesticación pero del hombre. La participación de todos los procesos tecnológicos en la vida del hombre, le han permitido a la industria avances inimaginables, acallando todo proceso de libertad crítica y de expansión, que le permita a cada individuo expresar sus ideas, pensamientos y sentimientos. Marcuse (1954, p. 35).

El impacto del progreso, convierte a la razón en sumisión. Debido a esto, la alienación racional y crítica del hombre no se ha hecho esperar. Supone que: "el hombre unidimensional, surge de acuerdo a toda aquella manipulación y adoctrinamiento a la 
que ha sido sometido por la comercialización de productos que hacen parte de las falsas necesidades del individuo". Marcuse (1954, p. 47).

Los nuevos sistemas tecnológicos en cuanto a su desarrollo, han hecho que la razón del hombre se pierda más rápido en cuanto a su manera de pensar, mientras más sentimientos de culpa exprese, peor se sentirá en todas sus dimensiones. Por esto, el hombre seguirá siendo unidimensional, o sea, en una sola dimensión, en un solo sentido: alienado.

Marcuse enumera unos factores importantes y lo que han producido en él:

- La mecanización del trabajador.

- La estratificación en la escala ocupacional.

- La modificación en su actitud y su conciencia.

- El debilitamiento como trabajador. Por esta razón, Marcuse decía que el gran problema del ser humano se debe a la automatización a la que ha sido sometido por este sistema. La máquina se convirtió en el alma y señor del mundo industrializado.

Continúa Marcuse haciendo una serie de planteamientos en cuanto a la situación del hombre y en lo que se ha convertido, debido a la forma como la sociedad está manejando los hilos conductores que lo están llevando a no conseguir el bienestar que quiere, sino antes por el contrario, lo están perdiendo en todas sus dimensiones. Marcuse (1954, p. 68).

De sí los profesores tenemos pensamiento crítico, nos tensiona González (2017) que:
Los profesores no enseñamos pensamiento crítico sino un pensar parametralizado, no enseñamos claves de humanidad por caer en racismos, no enseñamos a incluir sino a excluir, no enseñamos a ser diversos sino a exigir que el otro se homogenice, no enseñamos el sentido común sino altos valores que nadie práctica, no enseñamos a practicar deportes sino a seguir unos clubes de fútbol para hacernos violentos, y lo peor, no enseñamos a construir lenguajes propios sino a seguir replicando los diccionarios de los poderes que nos han colonizado. (p. 243)

Es un desafío, una insistencia que nos conmueve identificar el pensamiento crítico en los profesores.

\section{Ruta Crítica}

Para explicar la metodología empleada en la realización de este trabajo de investigación, necesitamos precisar los medios que nos permitieron llegar a definir los objetivos del mismo. Los presentamos así:

- Auto-biografías o correlatos de cada uno de los integrantes de este proyecto.

- Diálogo con autores representativos del pensamiento crítico.

- Consulta de tesis relacionadas con las dimensiones del pensamiento crítico.

- Descripción de las principales características del pensamiento crítico.

- Diálogo, discusión y comentarios de los integrantes, en los encuentros de investigación, como en los Módulos y Seminarios de la Maestría. 
Mostramos las evidencias del proceso realizado, presentando algunos fragmentos de los correlatos $\mathrm{o}$ auto-biografías de los integrantes del equipo:

En relación a los aspectos relacionados con la episteme y el diálogo con los teóricos, "este análisis crítico de mi vida, me ha permitido darme cuenta de mi pensar crítico, como lo menciona McLaren en la pedagogía crítica y la pedagogía revolucionaria $y$, en mis clases iniciar como consecuencia de la apropiación de esa nueva forma de pensar, mediante la apropiación y aplicación de las didácticas no parametrales". González, Henry (2014).

El compromiso del maestro como palabra dinamizadora, "me ha permitido lograr a través de la Maestría en educación, aplicar algunos conceptos aprendidos, a mis grupos de clases en el Politécnico Colombiano Jaime Isaza Cadavid: también debo reconocer que hay una serie de conceptos que he tenido que consultar, analizar, reflexionar, con el fin de elaborar su contenido, por ejemplo, ¿cuándo se puede decir que una persona aprendió algo? ¿Entonces: qué es aprender? Y por lo tanto, ¿qué es enseñar, qué tengo qué enseñar y cómo debo enseñarlo para que el alumno aprenda?" Botero (2014).

Aspectos importantes de la emoción y del afecto de otro de los investigadores, se manifiestan en: "en una ocasión, mi hija estaba bailando, la niña se cayó hacia atrás, se golpeó la cabeza y perdió el sentido por minutos, pudo haber sido que al caer, le produjo vómito que no lo expulsa, sino que se le va al pulmón y éste no le envía oxígeno al cerebro, lo cual ocasiona su muerte. Mi hija fue enterrada en Jardines de la $\mathrm{Fe}$, cementerio ubicado en Bello, Antioquia, Colombia, momentos que se pueden describir, pero con mucho dolor, que es preferible no recordar". Correa (2014).

Otro de los investigadores expresa su rebeldía de la siguiente forma: "esta, ha sido una de mis grandes rebeldías, nunca me ha gustado que me condicionen y en los diferentes estudios que he realizado, es con lo primero que uno se encuentra: el condicionamiento. Hoy, estamos condicionados por todo, desde que nacemos hasta que morimos y nunca he estado de acuerdo con esa postura". Zambrano (2014).

También se realizaron varios encuentros de investigación, donde cada grupo socializaba lo que había hecho hasta el momento y al final a cada equipo le hacían las devoluciones, comentarios, correcciones, modificaciones y retro-alimentaciones de sus proyectos.

En diferentes encuentros de nuestro equipo de investigación, realizados en el Politécnico Jaime Isaza Cadavid de Medellín, en la Universidad de Manizales, en la ciudad de Neiva, Huila, Colombia, con otros maestrantes $y$ en distintos períodos de tiempo, se lograron definir y determinar una serie de palabras fuerza, que fueron dinamizadoras del trabajo, desde los correlatos, desde la pregunta de investigación, nos permiten demostrar las evidencias de nuestro 
trabajo, a través de las vivencias antes mencionadas de cada uno de los investigadores. Ellas son: Emoción, Compromiso, Sentimientos, Visión, Rebeldías, Servicio, Responsabilidad y Emancipación.

Posteriormente se obtuvo información relacionada con el tema a investigar, por parte de otros investigadores que tuviesen conocimiento del tema, que hubiesen presentado artículos o ponencias sobre el mismo y que estuvieran vinculados con la docencia. (los cuales fueron definidos por cada uno de los investigadores) Se definió la finalidad de la maestría en educación como una "construcción de la re-significación de la docencia".

\section{Confluencias}

Entendemos las confluencias como los aspectos con los cuales nos identificamos en el equipo de trabajo, cuando nos referimos al significado, análisis y aplicación del pensamiento crítico, en los diferentes contextos o ámbitos donde se puede considerar. Para lograr expresar estas confluencias, el equipo de trabajo después de varias reuniones y entrevistas, se dedicó a definir, comentar y discutir los diferentes tópicos que encontramos en los correlatos de los investigadores y en los diálogos de cada uno de los integrantes del equipo con los teóricos de la Escuela de Frankfurt, los cuales fueron escogidos aleatoriamente para consultarlos, como también a Francisco Mora, en sus análisis, pensamientos e ideas sobre la neuro-educación, los cuales ya mencionamos en la pá- gina 9 en el capítulo de "Conversando con los teóricos"

Lo anterior, nos permitió estructurar unas ideas pertinentes que contribuyeron a precisar y construir los aportes que vamos a mencionar:

- El docente con pensamiento crítico motiva al estudiante a la participación en los diferentes contextos, desde su niñez hasta la vida adulta.

- El docente con pensamiento crítico, no debe sentir temor a expresar lo que piensa, siente, o quiere hacer.

- El docente con pensamiento crítico, debe saber identificar el aspecto histórico de su enseñanza, teniendo en cuenta el entorno y el medio donde desarrolla su proceso de aprendizaje.

- El docente con pensamiento crítico, comprende los cuestionamientos que hace la sociedad sobre el papel que posee la comunicación, por la desigualdad en el poder y por la creación de una industria cultural.

- El docente con pensamiento crítico, identifica la crítica al "consumismo" como algo que no hace al hombre plenamente feliz.

- El docente con pensamiento crítico, es capaz de entender las dimensiones políticas, económicas y sociales que están influyendo en la sociedad y concretamente, en el capitalismo, neoliberalismo, en la concentración de la riqueza y, como consecuencia, en el aumento de la pobreza.

- El docente con pensamiento crítico posee capacidad de discernimien- 
to, de inferencia lógica y de entendimiento, por el sometimiento que han tenido las personas, en la comercialización de sus productos y servicios.

- El docente con pensamiento crítico es consciente de que la calidad de la educación se mejora, a través del cambio en las prácticas didácticas y pedagógicas y de su participación en la transformación en el entorno que rodea a los sujetos.

\section{Desemejanzas}

Las desemejanzas hacen alusión a las diferencias en las vivencias, actuaciones, situaciones, estilos de vida, conflictos y problemas que haya vivido cada uno de los miembros del equipo de investigación, sus estudios, sus trabajos, sus familias, sus culturas, sus orígenes, sus amigos y cada una de las circunstancias que hayan sido importantes y relevantes en los momentos de vida de cada uno, lo cual se evidencia en los correlatos de los investigadores y esto lo construimos en diferentes encuentros, tal como lo manifestamos en el punto anterior.

- El enfoque filosófico, religioso, espiritual y académico de cada uno, es completamente diferente.

- La metodología utilizada para ofrecer las clases es diferente en el equipo, porque algunos manejábamos la educación tradicional.

- Los diferentes puntos de vista de cada uno, en cuanto a lo humano, cultural, social y político, hacen que lo académico sea distinto en cada uno de nosotros.
- La manera de abordar lo referente a la formación familiar, social y laboral, hacen que la forma de pensar del equipo, sea muy diferente.

- Las diferencias de criterios, en cuanto al análisis de los puntos esenciales del trabajo, se han manifestado a lo largo del proceso, pero se ha logrado llegar a una conciliación en los aspectos cruciales.

- Los conceptos de cada uno, en relación a la educación, a lo familiar, lo económico y lo político, dificultaron en algunos momentos el proceso y la realización del trabajo, en cuanto a los cambios de paradigmas mentales.

- La posición de nosotros, en cuanto a lo que pensamos y actuamos, se manifiesta de forma diferente, cada uno quiere defender su razonamiento personal.

\section{Hallazgos}

Queremos mencionar los aspectos encontrados en el trabajo de investigación y que fueron relevantes para nosotros, los cuales deseamos compartir con otras personas, como resultado del proceso investigativo, donde se identificaron las palabras que le ofrecieron dinamismo a este proceso: emoción, sentimientos, sujetos, influencias, entre otras, las cuales son inherentes al ser y por las cuales tomaremos en primer lugar las ideas principales identificadas por el equipo y enfocadas por el director del proyecto investigativo, Miguel Alberto González, sobre el pensamiento crítico: 
- "El pensamiento crítico debe ser un estilo de vida. El docente que posee pensamiento crítico duda de lo que sabe, no busca imponer verdades, sino que debilita sus propios dogmas y es un auténtico desafío intelectual para el otro" González, Miguel (2013).

- "Lo más dinámico del pensador crítico es la duda profunda, el no creer verdades, sus propias verdades". González, Miguel (2013).

- El docente que anime a sus estudiantes a tener un pensamiento crítico, es aquel que transmite un lenguaje, donde el estudiante sea capaz de diferenciar lo que quiere para él y para los demás.

- El quehacer educativo debe ser un acto mediado por el lenguaje, donde se realice un diálogo integral entre el docente y el estudiante y donde puedan expresarse las diferentes formas de pensar. "No somos la totalidad de la racionalidad". Guarín (2012).

- El lenguaje que utilice el docente en el aula, debe ser el puente que integre lo sensible del conocimiento y su relación con los estudiantes: "el lenguaje es el puente entre el estudiante y el docente". Guarín (2012).

- El docente que desee progresar en cuanto al conocimiento del pensamiento crítico, debe tener una posición de sencillez y humildad, para reconocer que él no se las sabe todas y que los estudiantes en muchas ocasiones pueden saber más que él.

- El conocimiento que el docente proponga a sus estudiantes, debe ser algo compartido y construido con los estudiantes, porque de ninguna forma las verdades que posea el docente, son absolutas ni propias, mediante la puesta en escena en el aula de las didácticas no parametrales.

- Para avanzar y progresar en la aplicación del pensamiento crítico, el docente no se puede considerar dueño absoluto del conocimiento, porque no estaría implementando el concepto de transformación de la sociedad, a través de sus enseñanzas y de su actuación humana y racional.

- El docente que quiera proponer el desarrollo del pensamiento crítico en sus estudiantes, debe tener en cuenta el aspecto transformador del mundo, con el fin de que haya una mayor equidad y una mejor forma de vivir.

- El estudiante con pensamiento crítico debe caracterizarse por expresar lo que siente sin temor de ser rechazado, por los que se consideran tienen todo el conocimiento del mundo, con respeto y prudencia, sin herir susceptibilidades.

- Para ser un docente con pensamiento crítico se necesita re-pensar todo aquello que no nos deja crecer como sujetos en la sociedad donde vivimos y que nos permita cambiar nuestra forma de ser, pensar, actuar, hacer y sentir.

- Por último, "La maestría en Educación-Docencia de la Universidad de Manizales, nos permite seguir siendo los mismos, pero pensando de manera diferente" Guarín (2012) y sí que se llega a pensar 
de manera diferente, con esto del pensamiento crítico.

\section{Conclusiones}

El objetivo de este trabajo de investigación fue identificar las características que son necesarias y convenientes para que un docente desarrolle en su actuación pedagógica de enseñanza, el pensar crítico, con el fin de que sea capaz de influir en sus estudiantes para que ellos a su vez también lleguen a pensar críticamente. Sin embargo, esto no garantiza que por el simple hecho de que el maestro sea crítico en sus planteamientos, también lo sean sus estudiantes de la misma forma y puedan llegar a ser generadores de transformación social, fuera de las aulas de clase.

Esperamos con el presente artículo, invitar a aquellos que puedan leerlo para que le den continuidad al proceso y a la vez ir registrando los avances encontrados.

Presentamos a continuación lo que para nosotros son las conclusiones del presente trabajo de investigación, teniendo como referencia nuestra pregunta de investigación: ¿En qué consiste un docente con pensamiento crítico?

- Siendo el aula el escenario de encuentro del pensamiento, el conocimiento y el lenguaje que garantiza el aprendizaje, es allí, donde el docente vive o debe vivir su propia humanidad.

- El estudiante que salga de las aulas académicas con pensamiento crítico, es capaz de diferenciar lo que quiere para él y para los otros, puede aportar y contribuir a que una so- ciedad cambie en la medida que las nuevas generaciones, sean sujetos transformadores de pensamiento.

- Si el docente cambia en su forma de percibir el mundo, lo más probable es que encontrará en sus estudiantes mentes con posibilidades de transformar el pensamiento, con sentido crítico.

- El acto educativo debe ser un acto amoroso, teniendo como medio el lenguaje y el pensamiento crítico, otros dirían: relación docente estudiante, basada en el diálogo integral, el pensar diferente nos permite reconocer la múltiple forma de ser docentes desde el pensamiento, los diferentes horizontes del pensamiento, son los que generan las diversas formas de relacionarnos. "No somos la totalidad de la racionalidad" Guarín (2012).

- El lenguaje utilizado por el docente en el aula, debe ser el puente, el amarre, todo aquello que sea capaz de unir desde lo sensible hasta lo ineludible, en su relación con los estudiantes, que son su principal herramienta.

- Es conveniente que los docentes cambien sus paradigmas que no los dejan ver mejor las circunstancias, y por lo tanto, esto los condena a vivir en un mundo sin razón, en un estado de enajenación absoluta, por la falta de querer pensar diferente y de manera crítica.

- Es importante que el docente sea transformador de pensamiento, para que logre llegar a ser crítico y para que sus estudiantes puedan hacerse nuevos planteamientos, a 
razón del mismo y encuentren en éste, una nueva filosofía de aprender a pensar, a actuar de manera más racional y humana, pero comprometidos con la sociedad.

- Adoptar nuevas posturas que lleven tanto al docente como al estudiante, mirar desde sus respectivos ángulos lo que ocurre en su entorno, para poder participar de una forma más concreta en la transformación del mundo, donde haya más equidad, mejor forma de vivir, pero sobre todo, tener la posibilidad de expresar lo que se siente, sin temor al rechazo de aquellos que se creen con todo el conocimiento del mundo.

- Ser un docente con pensamiento crítico, es a la vez, un sentimiento de de-colonización, de dejar atrás todo aquello que no nos deja crecer como sujetos, de terminar con una servidumbre de siglos en cuanto a nuestra forma de pensar, ser, hacer, actuar y sentir.

\section{Navegando sobre las Recomendaciones}

Con las siguientes recomendaciones, queremos puntualizar los aspectos fundamentales que deben tenerse presentes, para mostrar la utilidad y aplicación de nuestro estudio de investigación, a las diferentes situaciones o ámbitos de la vida y concretamente lo que respecta a la educación, que es nuestro producto esencial a considerar.

Para comprender el significado de nuestras recomendaciones, nos permitimos comentar que no tiene sentido, realizar una serie de consultas, análisis, comentarios, discusiones, apropiaciones de contenido y estudio de los temas desarrollados, si no los ponemos al servicio de los demás, porque consideramos que, obtener un conocimiento sobre este tema y no darlo u ofrecerlo a otras personas, sería algo poco constructivo y poco conveniente para nosotros mismos, como para las Instituciones en las cuales estamos laborando, el Politécnico Colombiano Jaime Isaza Cadavid y la que nos ofreció esta magnífica oportunidad de mejorar nuestro rol, como lo es la Universidad de Manizales.

El acto pedagógico de enseñanza debe ser esencialmente una vocación y no simplemente una réplica de conocimientos, por eso, consideramos que el docente primero debe ser pedagogo con pensamiento crítico y no solo, transmisor de conocimientos. Nuestras recomendaciones son:

- Esta actitud del docente implica proceso, secuencia y disposición hacia la obtención del conocimiento y de sus múltiples implicaciones, de tal manera que a partir de la adopción del pensamiento crítico y puesta en práctica en el aula de didácticas no parametrales, se puedan realizar mejores desarrollos en las Instituciones educativas.

- Este trabajo final, es ante todo, un proceso de sensibilización y de adopción, que requiere de profesionales que quieran desarrollarse y progresar en su vida académica y personal, siguiendo las bases, producto de la investigación realizada, sobre qué es un docente con pensamiento crítico. 
- Si se quiere mejorar la calidad de la educación en las Instituciones educativas, y a nivel local, departamental o nacional, se necesita capacitar a los docentes en lo que a pensamiento crítico se refiere y al cambio de paradigmas, mediante el uso de didácticas no parametrales, con el fin de obtener un mejoramiento continuo en la educación.

- Lograr tener pensamiento crítico no es fácil, pero se puede generar el desarrollo de ideas que permitan investigar en los temas que se estén enseñando.

- Cuando encuentres que existen puntos de vista sobre un tema, que son diferentes al tuyo, consulta con otras personas, o con otros medios de información, en la biblioteca, o bases de datos.

En la siguiente ilustración, (№ 4,al final) nos permitimos hacer una síntesis de las principales características, aptitudes, cualidades, o elementos que definen y es conveniente que posea un docente con pensamiento críti$\mathrm{co}$, las cuales hemos recogido a lo largo de nuestro proceso de investigación.

Tener todos los atributos que mencionamos en la ilustración, no debe interpretarse como una utopía, o como algo inalcanzable, sino como un punto de reflexión que oriente la labor que realice un excelente maestro y que tenga entre sus necesidades y principales inquietudes, el pensar de manera crítica, para mejorar su labor pedagógica-docente-enseñanza.

Los 24 atributos, o elementos propuestos en la ilustración, podemos resumirlos en seis, que consideramos esenciales, pues los demás son complementarios de la labor docente. Estos atributos los presentamos así:

- Hábil en el pensar: es una de las principales características del pensador crítico, porque si no la posee, cómo va a realizar análisis, o juicios críticos.

- Creativo: cuando una persona es creativa, posee mayores posibilidades de realizar juicios críticos sobre las situaciones que vive.

- Analítico: contribuye a la creación del pensar crítico, en la medida que se analicen las problemáticas que deba resolver.

- Innovador: una de las cualidades del innovador es la de saber afrontar los cambios que se presenten, lo mismo que la del pensador crítico.

- Reflexivo: la capacidad de una persona para reflexionar lo que le sucede en su vida, la conduce a realizar juicios críticos sobre sus situaciones.

- Inteligente: se podría afirmar que es el atributo principal que integra los anteriores, porque permite la proximidad hacia el pensar crítico.

Los otros 18 elementos que aparecen en la ilustración, son complementarios de los anteriores y están más relacionados con aspectos de la personalidad del pensador crítico, como son: respetuoso, diligente, honesto, comprometido, proactivo, empático, solidario, atento, justo, equitativo, generoso, dialógico, entre otros.

Un docente con pensamiento crítico, debe enfocarse según los siguientes elementos: 
Figura 3: Dimensiones de un docente con pensamiento crítico

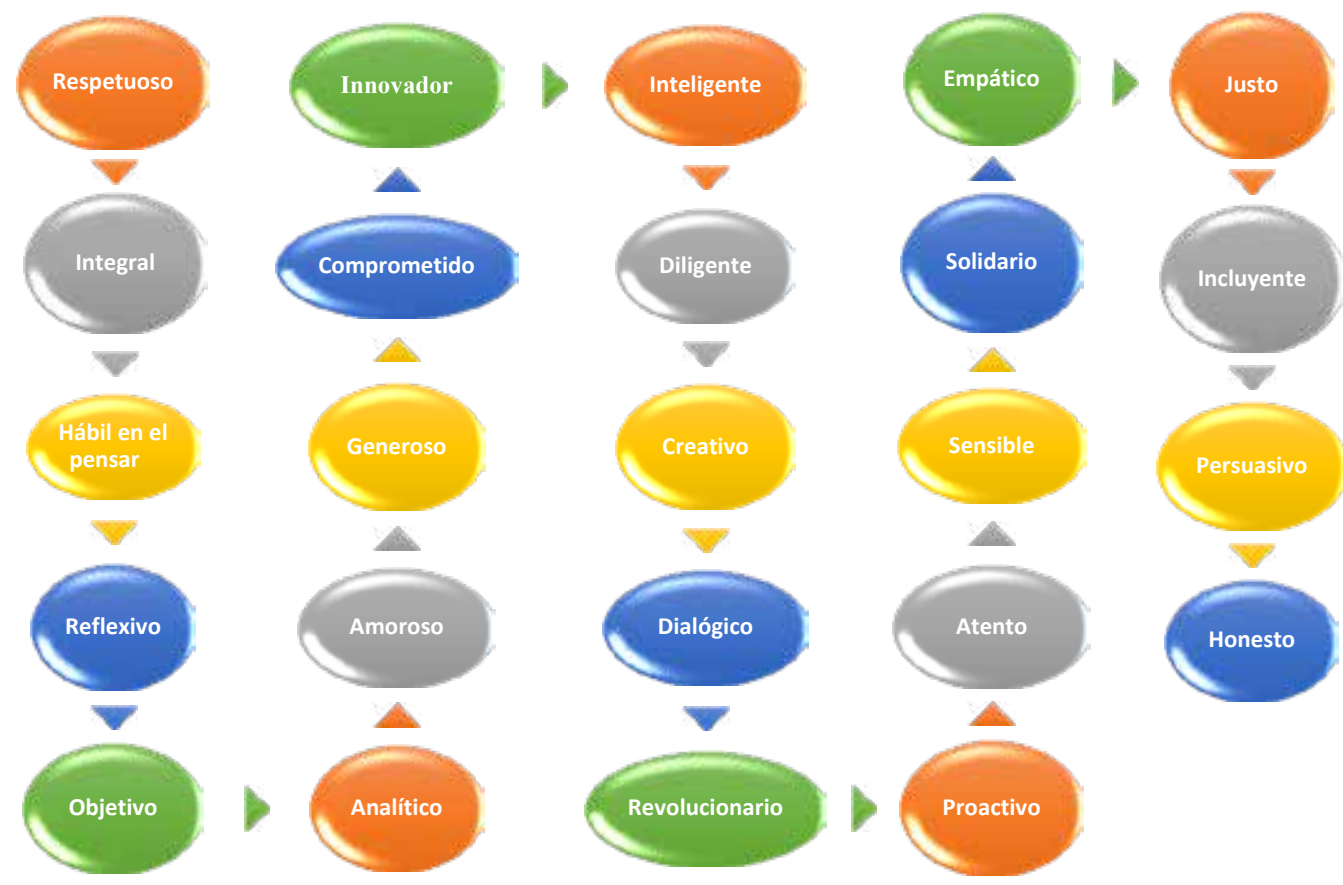

Fuente: Tomado de Botero, Correa, González, Zambrano (2014)

Bibliografía

Adorno Theodor et al. (1996). Pensamiento crítico: una declaración de consenso de expertos con fines de evaluación e instrucción. Asociación Filosófica Americana. Madrid: Tecnos.

Baudrillard, Jean. (2004). El Sistema de los Objetos. México: Siglo XXI.

Botero Álvarez, Juan Horacio. (20122014). Ensayos y reflexiones de su auto-biografía. Trabajo desarrollado para Maestría en Educación.

Correa López, Edgar. (2012-2014). La importancia del análisis del pensamiento crítico a partir de Héctor
Abad Faciolince. Aporte al trabajo de Maestría en Educación.

Frankl, Viktor. (1991). El hombre en busca de sentido. Barcelona: Herder. Freire, Paulo y Reglus, Neves. (2005). Pedagogía del Oprimido. México: Siglo XXI.

González Gaitán, Henry Helí. (20122014). El conocimiento a través de la Neuro-educación y Peter McLaren. Aportes en el análisis conceptual.

González G, M. A. (2017). Homogenizations-diversities and exclusions-inclusions in Colombian Education, captured through life 
stories | [Diálogos de saberes. las homogeneizaciones-diversidades $y$ las exclusiones-inclusiones en la Educación Colombiana, narrativas autobiográficas]. ISBN: 07989792. Revista de pedagogía, volume 38 , Isssue 103, 2017, pages 211-248

González G, M. A. (2010). Horizontes Humanos: Límites y Paisajes. Manizales: Universidad de Manizales. González G, M. A. (2012). Módulo Paisajes Escriturales. Manizales: Universidad de Manizales.

González G, M. A. (2010). Horizontes Humanos: Límites y Paisajes. Manizales: Universidad de Manizales.

González G, M. A. (2016). Miedos y olvidos peggógicos. Rosario: homosapiens.

González G, M. A. (2016). Un preludio de sorderas. Bogotá: Editorial Oveja negra.

Guarin Jurado, German. (2012). Modulo de Modernidad Critica. Manizales: Universidad de Manizales.

Habermas, Jürgen. (1981). Teoría de la Acción Comunicativa" Madrid: Taurus.

Hernández Sampieri, Roberto, Fernández Collado, Carlos y Baptista Lucio, Pilar. (2006). Metodología de la Investigación. 4a edición. México: McGraw-Hill.

Isaza de Gil, Gloria. (2013). Modulo Divesidad. Manizales: Universidad de Manizales.

Leclerc, Marc. (2007). De la Pedagogía Crítica a la Pedagogía de la Revolución: ensayos para comprender a Peter McLaren. México, Argentina, España: Siglo XXI.
Marciales Vivas, Gloria Patricia. (2004). Pensamiento crítico: diferencias en estudiantes universitarios en el tipo de creencias, estrategias e inferencias en la lectura crítica de textos. Tesis Doctoral.

Marcuse, Herbert. (1968). El Hombre Unidimensional. México: Joaquín Mortiz.

Mejia Valencia, Mario. (2013). Modulo Pedagogía Critica. Notas de clase. Manizales: CEDUM.

Menjura Escobar, Maria Ines. (2014). Modulo Horizontes Humanos. Manizales: Universidad de Manizales.

Mignolo, Walter. (2005). El pensamiento de-colonial, desprendimiento y apertura: un manifiesto. México: Siglo XXI editores.

Mora, Francisco. (2013). Neuro Educación: solo se puede aprender aquello que se ama. Madrid: Alianza Editorial.

Pamuk, Orhan Ferit. (2011). El Museo de la Inocencia. Barcelona: De Bolsillo.

Restrepo Garcia, Paula. Andrea. (2013). Módulo: Educación para la diversidad. Manizales: Universidad de Manizales.

Ríos Patiño, Ana. Gloria. (2014). Modulo Teoría curricular. Manizales: Universidad de Manizales.

Rodríguez Castrillón, Wilman Alberto, Serna, Arles Fredy y González González, Miguel Alberto. (2014). Modulo Análisis de información. Manizales: Universidad de Manizales.

Rueda, Restrepo Nelson. (2006). Sistema para determinar las competencias complejas de los directivos de la I.E.S. Tesis presentada para 
optar al grado de Doctor en Ciencias de la Educación, MBA.

Terol Reig, Vicent. (2011).Lasinfluencias orientales en el pensamiento occidental. Universidad de Valencia, España.

Valerio Echavarría, Carlos. (2013). Modulo Análisis del Discurso: Perspectivas Teóricas y Metodo- lógicas. Manizales: Universidad Manizales.

Zambrano Berdugo, Alfredo Enrique. (2012-2014). La influencia del hombre unidimensional en el pensamiento crítico Aportes al trabajo organizativo del informe final.

Zemelman, Hugo Hernan. (2009). Uso critico de la teoría. Mexico: IPN. 